\title{
Predictive models of syncope causes in an outpatient clinic
}

\author{
D. Graf ${ }^{a}$, J. Schlaepfer ${ }^{\mathrm{a}}$, E. Gollut ${ }^{\mathrm{a}}$, G. van Melle ${ }^{\mathrm{b}}$, C. Mischler ${ }^{\mathrm{a}}$, M. Fromer ${ }^{\mathrm{a}}$, \\ L. Kappenberger ${ }^{a}$, E. Pruvot ${ }^{a, *}$ \\ a Service de Cardiologie, Centre Hospitalier Universitaire Vaudois, BH07, CHUV, Rue du Bugnon 46, 1011 Lausanne, Switzerland \\ ${ }^{\mathrm{b}}$ Institut de Médecine Sociale et Préventive, Centre Hospitalier Universitaire Vaudois, 1011 Lausanne, Switzerland
}

Received 29 May 2006; received in revised form 3 November 2006; accepted 11 December 2006

Available online 29 March 2007

\begin{abstract}
The investigation of unexplained syncope remains a challenging clinical problem. In the present study we sought to evaluate the diagnostic value of a standardized work-up focusing on non invasive tests in patients with unexplained syncope referred to a syncope clinic, and whether certain combinations of clinical parameters are characteristic of rhythmic and reflex causes of syncope.

Methods and results: 317 consecutive patients underwent a standardized work-up including a 12-lead ECG, physical examination, detailed history with screening for syncope-related symptoms using a structured questionnaire followed by carotid sinus massage (CSM), and head-up tilt test. Invasive testings including an electrophysiological study and implantation of a loop recorder were only performed in those with structural heart disease or traumatic syncope. Our work-up identified an etiology in $81 \%$ of the patients. Importantly, three quarters of the causes were established non invasively combining head-up tilt test, CSM and hyperventilation testing. Invasive tests yielded an additional 7\% of diagnoses. Logistic analysis identified age and number of significant prodromes as the only predictive factors of rhythmic syncope. The same two factors, in addition to the duration of the ECG P-wave, were also predictive of vasovagal and psychogenic syncope. These factors, optimally combined in predictive models, showed a high negative and a modest positive predictive value.

Conclusion: A standardized work-up focusing on non invasive tests allows to establish more than three quarters of syncope causes. Predictive models based on simple clinical parameters may help to distinguish between rhythmic and other causes of syncope.
\end{abstract}

(C) 2007 Elsevier Ireland Ltd. All rights reserved.

Keywords: Syncope; Diagnostic yield; Diagnosis; Syncope unit

\section{Introduction}

Syncope, a common and challenging symptom, remains unexplained in up to $60 \%$ of the cases [1-6]. Some investigators have recently shown the superiority of simple investigation strategies over usual practice in patients with syncope addressed to emergency departments $[1,6-8]$. The development of syncope clinics has dramatically changed the evaluation of syncope by re-orienting patients toward functional investigations; however, little is known about the

\footnotetext{
* Corresponding author. Tel.: +41 21 3140104; fax: +41 213140013.

E-mail address: etienne.pruvot@chuv.ch (E. Pruvot).
}

true diagnostic performance of these dedicated facilities. Moreover, patients with syncope often present with multiple symptoms before and/or after the event. Syncope-related symptoms have been traditionally used to separate vasovagal from rhythmic causes [9-11] but predictive models of syncope causes are still lacking.

In the present work we investigated a population of patients referred to a syncope unit for unexplained syncope. We sought first to evaluate the diagnostic yield of a standardized work-up, which turned out to correspond closely to later published guidelines [12], and second, whether a certain combination of clinical parameters based on history, ECG and syncope-related symptoms were characteristics of either rhythmic or reflex causes of syncope. 


\section{Materials and methods}

\subsection{Setting}

This single center study was conducted in the syncope clinic of the Service of Cardiology at the Lausanne University hospital in Switzerland. On average, one patient is referred daily to the clinic for the investigation of unexplained syncope or presyncope. The study complies with the Declaration of Helsinki, and has been approved by our local ethics committee. Syncope was defined as a brief, self-limited loss of consciousness with the inability to maintain postural tone. Presyncope was defined as a near syncopal event. Patients with symptoms compatible with other non syncopal conditions such as seizure disorders, vertigo, dizziness or coma were excluded.

\subsection{Study design}

Consecutive outpatients referred to our syncope clinic were prospectively included between December 1st 1999 and October 30th 2001. Patients underwent a standardized workup (Fig. 1) consisting of a history, physical examination and 12-lead ECG analyzed by two of the investigators (E.P. and E.G.). Patients underwent a 30-min head-up tilt test (HUT) followed by upright and supine CSM in absence of contra-indications [12], with continuous non invasive blood pressure measurement (Finapres, Ohmeda). Drug challenges with intravenous adenosine triphosphate [13] and sublingual dinitrate isosorbide [14] were performed following a negative baseline HUT and CSM. Hyperventilation testing was performed only in patients with phobic, anxious and/or depressive features. Evaluation by a psychiatrist was required in all clinically suspect cases. Structural heart disease was ruled out on the basis of history, physical examination and ECG [12]. When the initial evaluation confirmed or suggested an underlying cardiac disease, a stress test and an echocardiogram were usually performed. The latter was also performed before any invasive study in patients not previously eval-

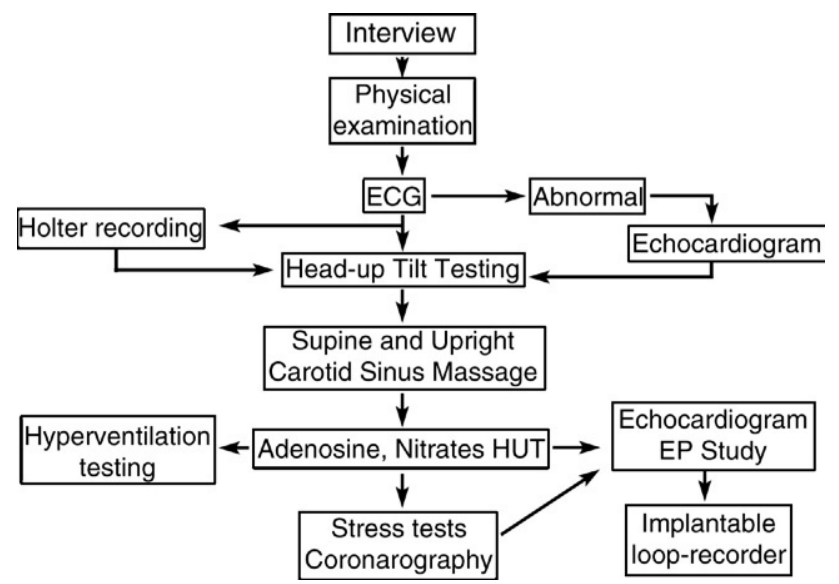

Fig. 1. Standardized work-up. uated. Patients then re-integrated the common work-up. Coronary angiography was performed when indicated. Electrophysiological (EP) study was performed only in patients with an underlying structural heart disease, or in those whose non invasive work-up was negative but who required further testing because of major trauma and/or for medico-legal purposes. Importantly, a positive test was considered diagnostic when the test-induced symptom(s) matched the presentation of the clinical syncope, otherwise the test was considered abnormal but non diagnostic.

\subsection{Measurements}

In order to maximize the reliability of data acquisition, physicians in charge of the patients were trained to use a 600 items database (FileMaker Pro 5) specifically developed for the management of syncope patients. The initial interview focused on the number of syncopal episodes, precipitating factors, occurrence and duration of prodromal and recovery symptoms. The following 23 symptoms were systematically investigated: diaphoresis, nausea and/or vomiting, visual changes, dyspnea, headache, chest pain, abdominal pain, palpitations, vertigo, asthenia, incontinence, neurologic deficit, impression of imminent death, diarrhea, sudation, tinnitus, paresthesia, anxiety, tongue biting, difficulty to concentrate, confusion, disorientation and tremor. Syncope-related trauma was classified as: (1) major, defined as any fractures, head injury or internal organ damage, or syncope resulting in a car accident; or (2) minor, defined as any bruise, cut, or soft tissue injury. The duration of the P-wave of the ECG was determined by averaging the $\mathrm{P}$-wave value from three consecutive beats, from at least two derivations (D2 and V1) [15]. Importantly, P-wave duration was measured and reported in a database before any investigations in order to avoid methodological bias.

\subsection{Diagnostic criteria for causes of syncope}

Diagnostic criteria for causes of syncope were established before the study and adhered strictly to published data [12]. The diagnosis was assigned by one of the investigators (E.P.) at the end of the standardized work-up which turned out to follow the guidelines of the European Society of Cardiology [12]. We did not establish final diagnoses on history alone or as a part, but always using results of investigational tests. Prodromes (i.e. history) were only used to establish that the syncopal event taking place during a specific test did reproduce the clinical event. However, the nature of prodromes (i.e. nausea, diaphoresis, etc) in itself was not used to establish final diagnoses.

For statistical analysis, final causes of syncope were grouped into 5 categories: (1) Rhythmic causes included bradyarrhythmias (AV block and cardio-inhibitory carotid sinus syndrome, CSS) and tachyarrhythmias (supra- and ventricular tachycardia); (2) VV/Psy causes included vasovagal (VV, i.e. tilt induced) syncope and psychogenic pseudo- 
Table 1

Baseline characteristics of the 317 patients

\begin{tabular}{ll}
\hline Age - years & $53 \pm 20^{1}$ \\
Female & $146(46)$ \\
Hypertension & $114(35)$ \\
Hypercholesterolemia & $90(28)$ \\
Diabetes type 2 & $20(6)$ \\
Active smoker & $78(25)$ \\
Coronary artery disease & $54(17)$ \\
Syncope frequency & $6 \pm 17^{1}$ \\
Presyncope only & $42(13)$ \\
Time elapsed since first episode _ years & $5 \pm 8^{1}$ \\
Minor trauma & $68(21)$ \\
Major trauma & $50(16)$ \\
Hospitalization & $198(62)$ \\
\hline
\end{tabular}

${ }^{1}$ Mean \pm SD, unmarked data are $n(\%)$.

syncope (Psy); (3) Hypotensive causes included orthostatic hypotension and vasodilatative CSS; (4) Miscellaneous, and (5) Undetermined when none of the above was applicable at the end of the standardized work-up. The latter three categories will be referred as Others in the sequel.

\subsection{Statistical analysis}

Analysis and construction of predictive models were based on the first 317 consecutive patients included in the study between December 1999 and October 2001. Validation of the model was done on the next 65 consecutive patients who met the inclusion criteria. For the construction of the model a univariate screening of the different variables was performed using Fisher's exact test to compare proportions of any specific factor in the three main categories of syncope, namely Rhythmic, VV/Psy or Others (see text for definitions). Thus, in addition to age, sex, blood pressure and other history parameters, 23 symptoms were tested and the significance level was set at 0.001 to protect for the multiplicity of tests (Bonferroni). Continuous variables were dichotomized for simplicity and reproducibility, except age, which was categorized in five (quintiles) or three (tertiles) in order to assess whether age effect could be taken as linear. It turned out that the quadratic component was not significant so that the linear trend is adequate.

Significant factors (at 0.001) in this univariate screening were then entered in a multivariable logistic regression and a stepwise selection (step down) was used to build the model (probability to remove 0.10 ). The overall significance of the model is based on the Likelihood Ratio test. Two models were constructed, one which attempted to characterize Rhythmic cases versus all Others, and another characterizing VV/Psy cases versus all Others. These regression results were then used to produce two so-called rules based on a simplified reading of the results. Thus, using the combination of these rules, patients could be classified as either probably Rhythmic, probably VV/Psy, possibly both or neither. The results of these rules have been confronted with the real data in Table 6A (construction sample of 317) and 6B (validation sample of 65).

\section{Results}

\subsection{Patient characteristics and causes of syncope}

During the study period 317 consecutive patients were enrolled. Table 1 reports the clinical characteristics of the study group (mean age $53 \pm 20$ y). The mean number of syncopal episodes per patient was 6 , with $13 \%$ of the patient referred for presyncope only. Syncope resulted in a major traumatic event in $16 \%$ and hospitalization in $62 \%$ of the patients. Table 2 describes the spectrum of causes as diagnosed after our standardized work-up. Vasovagal (tiltinduced) syncope was the most prevalent cause (23\%), followed by cardio-inhibitory CSS (18\%). The prevalence of psychogenic pseudo-syncope was surprisingly high $(n=55$, $17 \%$ ), with panic disorders in 52 patients and conversion disorder in 3 patients. Of these, half $(n=25,45 \%)$ manifested spontaneously during HUT, while the remaining diagnoses $(n=30,55 \%)$ were established during the hyperventilation test. In both cases, the diagnosis was established because the spontaneous or triggered response matched the clinical syncope. Hypotensive disorders (orthostatic and vasodilatative CSS) were diagnosed in $8 \%$ and tachyarrhythmic syncope in $7 \%$ of the patients. Other rare causes of syncope included hypertrophic obstructive cardiomyopathy and neurological causes (i.e. sub-clavian steal syndrome and seizure). Finally, an implantable loop recorder (ILR, Reveal, Medtronic) was proposed to 17 patients because of syncope-related complications; 13 patients were implanted and 4 refused the procedure. The device yielded a diagnosis in $6(46 \%)$ patients with an equal proportion of supraventricular tachycardia $(n=2)$, epilepsy $(n=2)$ and hypotension $(n=2)$. Interestingly, both patients with supraventricular tachycardia had a negative EP study, and the two patients diagnosed as epileptic had a prior negative electroencephalogram. Adding the 1.8\% of diagnoses yielded by ILR incremented the overall diagnosis rate of our standardized work-up from $79 \%$ to $81 \%$. Of note, removing psychogenic causes which did not

Table 2

Causes of syncope

\begin{tabular}{lc}
\hline & $n(\%)$ \\
\hline Neurally-mediated & \\
$\quad$ Vasovagal (tilt-induced) & $72(23)$ \\
Situational & $8(3)$ \\
Vasodilatative CSS & $14(4)$ \\
Cardio-inhibitory CSS & $56(18)$ \\
Psychogenic pseudo-syncope & $55(17)$ \\
Orthostatic & $10(3)$ \\
Cardiac arrhythmias & \\
Tachyarrhythmic & $22(7)$ \\
$\quad$ Ventricular tachycardia & $11(3)$ \\
$\quad$ Supraventricular tachycardia & $11(3)$ \\
AV block & $7(2)$ \\
Miscellaneous & $5(2)$ \\
Unexplained & $68(21)$ \\
Total & $317(100)$ \\
\hline
\end{tabular}

CSS: carotid sinus syndrome. 
Table 3

Diagnostic yield of current tests

\begin{tabular}{lc}
\hline & $n / N(\%)$ \\
\hline ECG & $4 / 317(1)$ \\
Orthostatic blood pressure test & $15 / 295(5)$ \\
Carotid sinus massage & $38 / 264(14)$ \\
Head-up tilt test & $92 / 266(35)$ \\
Hyperventilation test & $61 / 78(78)$ \\
Long term ECG recording & $11 / 95(12)$ \\
Echocardiogram & $4 / 187(2)$ \\
Exercise test & $8 / 107(7)$ \\
Electrophysiological study & $15 / 61(25)$ \\
Implantable loop recorder & $6 / 13(46)$ \\
\hline
\end{tabular}

$N=$ number of tests performed; $n=$ number of diagnostic tests.

manifest spontaneously ( $9 \%$ of final causes) reduced only moderately the overall diagnostic yield of the work-up (72\%).

\subsection{Diagnostic yield of current tests}

Table 3 reports the diagnostic yield of individual tests used to establish syncope causes. CSM was performed in 264 patients, with $36 \%$ abnormal responses and $14 \%$ diagnostic tests; nearly all (35/38) CSM were diagnostic in the upright position while fewer than half (16/38) were positive supine. HUT was performed in 266 patients with 36\% abnormal responses and $35 \%$ diagnostic tests. Of note, a psychogenic pseudo-syncope was spontaneously observed during HUT in an additional 22 patients. Hyperventilation testing was performed in 78 patients: it reproduced the clinical event in the 22 patients with a spontaneous psychogenic manifestation during HUT, and provided evidence for panic attacks as a cause for syncope in an additional 39 patients, of which 9 also had another diagnosis. Thus, the overall diagnostic yield of hyperventilation testing in patients with suggestive features at history or spontaneous manifestation during HUT achieved $78 \%$. The hyperventilation test triggered syncope or near syncope in $13(21 \%)$ of the 61 positive patients, and matched prodromal symptoms (without syncope or near syncope) in the remaining 48 (79\%) patients. EP study was diagnostic in $25 \%$ of the 61 tested patients. Importantly, $74 \%$ of the final causes of syncope were identified using non invasive testing, while invasive testings (EP study and ILR) yielded an additional $7 \%$ of diagnoses.

\subsection{Predictive rules of Rhythmic and VV/Psy categories of syncope}

Logistic analysis identified 11 prodromes of the 23 screened symptoms as significantly related to categories (i.e. Rhythmic and VV/Psy) of syncope. Table 4 reports the distribution of the 11 significant prodromes in the 5 categories of syncope causes as defined in the Materials and methods section. Interestingly, absence of prodromes (i.e. sudden syncope) was much more prevalent in Rhythmic than in the other defined causes. Conversely, nausea/vomiting, diaphoresis, sudation, paresthesia and palpitations were much more prevalent in VV/Psy causes than in any other category, except for Miscellaneous which shared some common features. Hypotensive causes showed no distinctive pattern, while Undetermined causes shared some similarities with Rhythmic causes with a high prevalence of sudden onset.

Logistic regression identified age of the patients and number of prodromes (among the 11 significant ones) as the

Table 4

Distribution of statistically* significant parameters in final categories of syncope

\begin{tabular}{|c|c|c|c|c|c|c|}
\hline \multirow[t]{3}{*}{ Prodromes } & Rhythmic & VV/Psy & Нypo & Miscellaneous & Unexplained & Total \\
\hline & $N=85$ & $N=127$ & $N=24$ & $N=13$ & $N=68$ & $N=317$ \\
\hline & $n(\%)$ & $n(\%)$ & $n(\%)$ & $n(\%)$ & $n(\%)$ & $n(\%)$ \\
\hline Age — years & $68 \pm 12$ & $40 \pm 17$ & $68 \pm 16$ & $60 \pm 16$ & $56 \pm 18$ & $53 \pm 20$ \\
\hline P-wave duration $-\mathrm{ms}$ & $115 \pm 15$ & $101 \pm 12$ & $117 \pm 18$ & $108 \pm 13$ & $109 \pm 15$ & $108 \pm 16$ \\
\hline Number of prodromes & $1 \pm 2$ & $4 \pm 2$ & $2 \pm 2$ & $2 \pm 1$ & $1 \pm 2$ & $3 \pm 2$ \\
\hline None & $41(48)$ & $11(9)$ & $2(8)$ & 0 & $37(54)$ & $91(29)$ \\
\hline Nausea/vomiting & $8(9)$ & $47(37)$ & $1(4)$ & $3(23)$ & $8(12)$ & $67(21)$ \\
\hline Diaphoresis & $12(14)$ & $60(47)$ & $5(21)$ & $4(31)$ & $11(16)$ & $92(29)$ \\
\hline Sudation & $9(11)$ & $56(44)$ & $6(25)$ & $4(31)$ & $10(15)$ & $85(27)$ \\
\hline Blurred vision & $13(15)$ & $69(54)$ & $11(46)$ & $4(31)$ & $17(25)$ & $114(36)$ \\
\hline Paresthesia & $1(1)$ & $36(28)$ & $1(4)$ & 0 & $2(3)$ & $40(13)$ \\
\hline Palpitations & $4(5)$ & $38(30)$ & 0 & 0 & $7(10)$ & $50(16)$ \\
\hline Vertigo/dizziness & $29(34)$ & $73(57)$ & $11(46)$ & $7(54)$ & $13(19)$ & $133(42)$ \\
\hline Dyspnea & $8(9)$ & $28(22)$ & $3(13)$ & 0 & $3(4)$ & $42(13)$ \\
\hline Anxiety & $2(2)$ & $25(20)$ & $1(4)$ & 0 & $4(6)$ & $32(10)$ \\
\hline Asthenia/weakness & $20(24)$ & $56(44)$ & $9(38)$ & $4(31)$ & $11(16)$ & $100(32)$ \\
\hline Headache & 0 & $21(17)$ & $2(8)$ & $1(8)$ & $2(3)$ & $26(8)$ \\
\hline
\end{tabular}

$*_{p}<0.001$ in the comparison of Rhythm, VV/Psy and other three categories pooled.

Hypo: orthostatic hypotension and vasodilatative CSS; VV/Psy: vasovagal and psychogenic syncope; $N$ : number of patients in each category; $n$ : number (\%) of positive patients; Miscellaneous causes included situational; Rhythmic: bradyarrhythmias (AV block and cardio-inhibitory CSS) and tachyarrhythmias (supraand ventricular); Undetermined: syncope remaining unexplained after the standardized work-up. 
Table 5A

Results of the multivariable logistic regression: model for Rhythmic syncope

\begin{tabular}{|c|c|c|c|c|c|}
\hline \multirow{2}{*}{$\begin{array}{l}\text { Logit } \\
\text { estimates }\end{array}$} & \multicolumn{3}{|c|}{ Number of observations } & \multicolumn{2}{|l|}{$=317$} \\
\hline & \multicolumn{3}{|c|}{ Likelihood ratio test } & \multicolumn{2}{|c|}{$p<0.00001$} \\
\hline Rhythmic & Coefficients & Standard error & WaldP & $95 \% \mathrm{CI}$ & \\
\hline AgeCat & 1.142 & 0.208 & $<0.0005$ & 0.734 & 1.550 \\
\hline ProdCat & -1.330 & 0.342 & $<0.0005$ & -2.002 & -0.659 \\
\hline Constant & -3.068 & 0.537 & $<0.0005$ & -4.120 & -2.015 \\
\hline
\end{tabular}

AgeCat and ProdCat are defined in the text and repeated in Table 5C. WaldP is the individual significance of each factor; CI: confidence interval. Coefficients on AgeCat and ProdCat suggest opposite effects for these factors (similar size but opposite sign); the resulting rule is shown in Table 5C.

only two significant factors predictive of Rhythmic causes. The same two factors, in addition to the "ECG P-wave duration", were also predictive of VV/Psy causes. For the logistic regression, age was categorized (AgeCat) into three classes scored: 1 for age ( $\leq 45 \mathrm{y} ; 2$ for age $45<X<65$ y and 3 for age $>65 \mathrm{y}$; similarly, prodromes were categorized into 2 classes (ProdCat) scored 0 for $\leq 1$ symptom, and 1 for $\geq 2$ symptoms. Finally the ECG P-wave duration was separated (P-waveCat) into two classes scored 0 for duration $<120 \mathrm{~ms}$ and 1 for duration $\geq 120 \mathrm{~ms}$ or non sinus rhythm (i.e. atrial fibrillation and flutter). AgeCat and ProdCat were optimally combined in a predictive model of Rhythmic syncope as follows: Rhythmic score $=$ AgeCat - ProdCat -2 ; a score $\geq 0$ classified patients as suffering from Rhythmic syncope, and a score $<0$ as non Rhythmic syncope. Similarly, AgeCat, ProdCat and P-waveCat were optimally combined in a predictive model of VV/Psy syncope using the following: VV/ Psy score $=2 \times$ ProdCat - AgeCat $-\mathrm{P}$-waveCat +2 ; a score $\geq 0$ classified patients as suffering from VV/Psy syncope, and a score $<0$ as non VV/Psy syncope. Tables 5A, 5B and $5 \mathrm{C}$ summarize predictive factors, rules and cut-off values for both Rhythmic and VV/Psy predictive models. For example a $40 \mathrm{y}$ old patient with syncope preceded by diaphoresis and sudation, and a normal $\mathrm{P}$-wave duration $(<120 \mathrm{~ms})$ has a Rhythmic score of $0(1-1-2=-2)$, which classifies the patient as not Rhythmic, and a VV/Psy score of $1(2 \times 1-0-1+2=3)$ which classifies the patient as VV/Psy.

Table 6A shows the joint classification using Rhythmic and VV/Psy models together. Forty nine percent of the

Table 5B

Results of the multivariable logistic regression: model for $V V / P s y$ syncope

\begin{tabular}{|c|c|c|c|c|c|}
\hline \multirow{3}{*}{$\begin{array}{l}\text { Logit estimates } \\
\text { VV/Psy }\end{array}$} & \multicolumn{3}{|c|}{ Number of observations } & \multirow{2}{*}{\multicolumn{2}{|c|}{$\begin{array}{l}=297 \\
p<0.00001\end{array}$}} \\
\hline & \multicolumn{3}{|c|}{ Likelihood ratio test } & & \\
\hline & Coefficients & Standard error & WaldP & $95 \% \mathrm{CI}$ & \\
\hline AgeCat & -1.194 & 0.223 & $<0.0005$ & -1.630 & -0.758 \\
\hline ProdCat & 1.960 & 0.312 & $<0.0005$ & 1.349 & 2.572 \\
\hline P-waveCat & -0.880 & 0.376 & 0.019 & -1.616 & -0.143 \\
\hline Constant & 1.142 & 0.445 & $<0.0005$ & 0.271 & 2.014 \\
\hline
\end{tabular}

The coefficient on ProdCat is roughly twice that of AgeCat and P-waveCat but with opposite sign; the resulting rule is shown in Table 5C. Only 297 patients had P-wave measurements; missing $\mathrm{P}$-waves (i.e. $\mathrm{AF}$ ) were assumed to have duration $\geq 120 \mathrm{~m}$.
Table 5C

Predictive rules of Rhythmic and VV/Psy syncope

\begin{tabular}{llll}
\hline & Predictive factors & Rules & Cut-off values \\
\hline Rhythmic & $\cdot$ Number of & AgeCat-ProdCat -2 & $\geq 0=$ Rhythmic \\
& prodromes & & \\
VV/Psy & $\cdot$ Age & & $<0=$ Not Rhythmic \\
& $\cdot$ Number of & $2 \times$ ProdCat - P- & $\geq 0=$ VV/Psy \\
& prodromes & waveCat - AgeCat +2 & \\
& $\cdot$ Age & & $<0=$ Not VV/Psy \\
& $\cdot$ P-wave duration & & \\
\hline
\end{tabular}

AgeCat scores 1 for age $\leq 45 \mathrm{y} ; 2$ for age $45<X<65$ y and 3 for age $>65 \mathrm{y}$; ProdCat scores: 0 for $\leq 1$ symptom, 1 for $\geq 2$ symptoms; P-waveCat scores: 0 for P-wave $<120 \mathrm{~ms}, 1$ for P-wave $\geq 120 \mathrm{~ms}$ or non sinus rhythm.

patients classified as Rhythmic were true Rhythmic with only $7 \%$ of true VV/Psy; $72 \%$ of the patients classified as VV/Psy were true VV/Psy with only 5\% of true Rhythmic. Importantly, none of the patients remained unclassified. Finally $58(18 \%)$ patients were classified as both Rhythmic and VV/Psy, of which $41 \%$ were true Rhythmic and $21 \%$ were true VV/Psy. These patients were ultimately considered as Rhythmic. Both predictive rules were then evaluated on the study population in a two-step classification model, and results were compared with final causes of syncope as given by the standardized work-up. Applying the Rhythmic model classified 166/317 (52\%) patients as Rhythmic with a sensitivity of $91 \%(77 / 85)$, a specificity of $62 \%(143 / 232)$, a positive predictive value (PPV) of $46 \%$ and a negative predictive value (NPV) of $95 \%$. Applying the VV/Psy model classified all remaining patients as VV/Psy with an overall sensitivity of $85 \%$ (108/127), specificity of $77 \%$ (147/190), PPV of $72 \%$ and NPV of $89 \%$. Predictive model for the other syncope causes were not developed because of the limited number of patients.

\subsection{Validation of the two rules}

The performance of the joint classification using both Rhythmic and VV/Psy models was then evaluated on a validation population of 65 newly included patients referred to the clinic. Applying the Rhythmic model classified 24/65 (37\%) patients as Rhythmic with a sensitivity of $59 \%$ (10/ 17), a specificity of $71 \%$ (34/48), a PPV of $42 \%$ and a NPV of

Table 6A

Classification based on the combination of Rhythmic and VV/Psy models in the derivation sample $(n=317)$

\begin{tabular}{llcc}
\hline & \multicolumn{2}{c}{ Rhythmic rule } \\
\cline { 3 - 4 } & & $<0=$ Not Rhythmic & $\geq 0=$ Rhythmic \\
\hline \multirow{2}{*}{ VV/Psy rule } & $<0=$ Not VV/Psy, $n$ & 0 & 108 \\
& True Rhythmic, \% & 0 & 49 \\
& True VV/Psy, \% & 0 & 7 \\
& True Others, \% & 100 & 44 \\
& $\geq 0=$ VV/Psy, n & 151 & 58 \\
& True Rhythmic, \% & 5 & 41 \\
& True VV/Psy, \% & 72 & 21 \\
& True Others, \% & 23 & 38 \\
\hline
\end{tabular}


Table 6B

Classification based on the combination of Rhythmic and VV/Psy models in the validation sample $(n=65)$

\begin{tabular}{|c|c|c|c|}
\hline & & \multicolumn{2}{|l|}{ Rhythmic rule } \\
\hline & & $<0=$ Not Rhythmic & $\geq 0=$ Rhythmic \\
\hline \multirow[t]{8}{*}{ VV/Psy rule } & $<0=$ Not VV/Psy, $n$ & 0 & 9 \\
\hline & True Rhythmic, \% & 0 & 66 \\
\hline & True VV/Psy, \% & 0 & 0 \\
\hline & True Others, $\%$ & 100 & 34 \\
\hline & $\geq 0=\mathrm{VV} / \mathrm{Psy}, n$ & 41 & 15 \\
\hline & True Rhythmic, \% & 17 & 26 \\
\hline & True VV/Psy, \% & 51 & 26 \\
\hline & True Others, $\%$ & 32 & 48 \\
\hline
\end{tabular}

$83 \%$. Applying the VV/Psy model classified all remaining patients as VV/Psy with an overall sensitivity of $84 \%$ (21/ $25)$, specificity of $50 \%(20 / 40)$, PPV of $51 \%$ and NPV of $83 \%$ (Table 6B).

\subsection{Meaning of P-wave duration}

We assessed the value of the $\mathrm{P}$-wave duration according to the categories of syncope. Interestingly, $14 \%$ (14/97) of the patients with prolonged P-wave duration were VV/Psy syncope while $42 \%$ (41/97) were Rhythmic syncope. In these 97 patients, the proportion of hypertension (53\%), ischemic heart disease (25\%) and NYHA class II (41\%) was high, with $70 \%$ having at least one of these clinical characteristics. In the 85 patients with Rhythmic syncope, the prevalence of hypertension (48\%), ischemic heart disease (27\%) and NYHA class II (45\%) was also high, as opposed to $12 \%, 5 \%$ and $10 \%$ respectively in the 127 patients with $\mathrm{VV} /$ Psy syncope.

\section{Discussion}

\subsection{Diagnostic yield of a standardized work-up}

The diagnostic approach of patients with syncope is a difficult and challenging clinical problem. Despite multiple investigations, syncope remains unexplained in $13-54 \%$ of the cases [1-6]. Diagnostic performance, however, varied widely between studies carried out with $[1,2,6,8,16,17]$ or without $[3,5,8]$ standardized work-up, in unselected $[1,5,6,16-18]$ or referred [3,19] patients. Early studies $[9,20,21]$ reported $50-63 \%$ of diagnoses in unselected patients; however, most of the causes were determined on the basis of clinical history and physical examination. Importantly, the superiority of standardized investigation strategies over usual practice has been recently shown in patients with syncope addressed to emergency departments [1,6-8,16-18]. Furthermore, the availability of syncope clinics has dramatically changed the daily practice of syncope by re-orienting patients toward functional tests such as HUT and CSM. The present study shows that a standardized work-up yielded an etiology in more than $75 \%$ of the patients, and that most causes were diagnosed non invasively combining HUT, CSM and hyperventilation testing. Importantly, syncope etiologies requiring invasive diagnostic procedures (i.e. tachyarrhythmias) remained rare and occurred in less than $7 \%$ of our population. Thus, similarly to Farwell et al. [17], the present study stresses the utility of a standardized work-up focusing on non invasive testing for the investigation of unexplained recurrent syncope referred to a syncope clinic.

\subsection{Distribution of syncope causes in a syncope clinic}

In the present study, the distribution of syncope causes is well in accordance with the published literature $[11,16,17,21,22]$. Vasovagal (tilt-induced) was the most prevalent (23\%), followed by cardio-inhibitory CSS (18\%), hypotensive (8\%) and tachyarrhythmic (7\%) syncope. Although the lack of gold standard remains a well known limiting factor for studies focusing on syncope, we paid careful attention to match final diagnoses with the clinical syncope. For instance, patients with typical tilt-induced vasovagal syncope but who complained of sudden syncope were not considered as vasovagal.

Psychogenic causes of syncope have been originally considered as negligible, accounting for less than $6 \%$ of etiologies $[6,16,20]$. Some studies [23-25], however, suggest that the prevalence of psychogenic pseudo-syncope may be as high as $26 \%$ in an unexplained syncope population. Importantly, Linzer et al. have shown the relationship between syncope and both panic and depression disorders [23,24]; the recurrence rate dropped from $50 \%$ in untreated patients to $10 \%$ in those undergoing a psychotherapy. The high prevalence of psychogenic pseudo-syncope (17\%) in the present study is at variance with recent studies performed in a similar set-up (syncope clinic) $[8,26]$. Our patients were systematically screened for anxiety, phobic and depressive features. Only those with positive feature(s) were tested at the end of our standardized work-up. Panic and conversion disorders were the most prevalent diagnoses. Panic disorders were diagnosed during hyperventilation testing (53\%) or spontaneous spells (42\%), while conversion disorders (5\%) always manifested spontaneously during HUT. During hyperventilation testing, only patients with symptoms strictly matching the clinical event were diagnosed as suffering from panic attacks. Moreover, other organic and reflexogenic causes of syncope had been previously excluded as part of our standardized work-up but a vasovagal event may still have occurred [27]. Interestingly, patients with psychogenic pseudosyncope were young $(40 \mathrm{y})$, with two thirds of female gender, and suffered more frequently of presyncope $(22 \%)$ than other better accepted causes such as vasovagal (13\%) or rhythmic (8\%) syncope. Removing psychogenic diagnoses without spontaneous manifestation from final causes still left $8 \%$ of our outpatient population with a non disputable diagnosis of psychogenic pseudo-syncope. In summary, the present findings suggest that panic, depressive and phobic features 
should be checked routinely in the evaluation of patients with unexplained syncope. Although a link between anxiety and vasovagal syncope has been suggested [28], further studies are needed to elucidate the pathophysiologic mechanisms leading to syncope.

Twenty one percent of the patients remained with unexplained syncope after our standardized work-up. Interestingly, these patients shared some features with Rhythmic patients ( $46 \%$ of sudden onset). An implantable loop recorder (Reveal, Medtronic) was proposed to 17 of these patients because of syncope-related complications; 13 patients were implanted and 4 refused the procedure. The device yielded a diagnosis in 6/13 (46\%) patients with an equal proportion of supraventricular tachycardia $(n=2)$, epilepsy $(n=2)$ and hypotension $(n=2)$. The present results confirm the incremental benefit of implantable loop recorder in patients with unexplained syncope after a negative standardized work-up [29].

\subsection{Predictive rules of Rhythmic and VV/Psy causes of syncope}

Some studies have evaluated the ability of clinical features to predict syncope causes [10,11,26,30,31]. Martin et al. reported that the duration of prodromal symptoms was longer for neurally-mediated than for cardiac syncope [11]. Calkins et al. reported that $\mathrm{AV}$ block and ventricular tachycardia frequently manifested as sudden syncope whereas vasovagal events were usually preceded by prodromal symptoms [10]. In contrast, Alboni et al. found that symptoms and signs considered suggestive of vasovagal syncope were equally distributed in both cardiac and neurally-mediated causes [26]. Recently, Sheldon et al. successfully predicted vasovagal causes using a simple point score in a syncope population recruited from primary hospitals and referral units [31]. Our findings extend some of their observations regarding the prevalence of symptoms and heart disease. Patients with non rhythmic syncope (vasovagal or psychogenic) reported multiple symptoms before the events, were younger and had little functional limitation and structural heart disease as compared to patients with brady- or tachyarrhythmic causes. Another intriguing finding of this analysis is that patients with vasovagal and psychogenic pseudo-syncope appeared to share many prodromal symptoms. Interestingly, Carey et al. have recently observed some hyperventilation-induced hypocapnia in the minutes preceding the onset of vasovagal syncope during head-up tilt test [32]. It is believed that hypocapnia is part of a counterregulation process to increase venous tone and preload before syncope, which might explain some of the common clinical features between vasovagal and psychogenic pseudo-syncope [32]. Alternatively, it may be that some patients classified as psychogenic are indeed suffering from vasovagal syncope.

In the present study, the positive predictive value of Rhythmic and VV/Psy models was modest, while both negative predictive values were high, which might be of clinical interest. Only the P-wave duration but no other ECG parameter (e.g. QRS and QT duration, Q wave, etc.) was identified by multivariable analysis as a predictor of syncope causes. Patients with prolonged P-wave duration were more likely to suffer from Rhythmic syncope including tachy- and bradyarrhythmias. Prolonged P-wave duration is a consistent finding in patients with sinus node disease [33] or ventricular arrhythmias, and could reflect some degree of electromechanical remodeling. In several studies, prolonged P-wave duration was shown to be a characteristic of patients with paroxysmal AF, and to be predictive of AF recurrence and failure to maintain sinus rhythm [34]. In addition, patients at very early stages of hypertension have demonstrable evidence of prolonged atrial conduction by P-wave signal-averaged ECG; also P-wave duration increased with severity of hypertension [35]. Interestingly, in patients with Rhythmic syncope P-wave duration $(115 \pm 15 \mathrm{~ms})$ was prolonged as compared to patients with VV/Psy syncope ( $101 \pm 12 \mathrm{~ms})$. In patients with Rhythmic syncope, the prevalence of hypertension, ischemic heart disease and congestive heart failure was higher than in patients with VV/Psy syncope. Thus prolonged P-wave duration identifies a subgroup of patients more likely to suffer from cardiovascular disorders and Rhythmic syncope.

\subsection{Limitations}

Our study bears some limitations. First, the present findings are hardly applicable at primary care emergency departments because patients were referred from a wide geographical area. Second, in the absence of a gold standard some uncertainty remains about the attribution of syncope causes, which is particularly true for psychogenic pseudosyncope. Attribution of a diagnosis, however, relied on the matching of patients' clinical event(s) by results of investigational tests, and followed strict diagnostic criteria [12]. Third, whether the standardization of syncope management is cost- and recurrence rate effective has not been addressed in the present study. Some recent studies using a similar set-up, however, suggested so [36,37].

In conclusion, the main findings of our study are that: (1) a standardized work-up yields more than three quarters of causes in patients referred to a syncope clinic for unexplained syncope, most causes being diagnosed using non invasive tests; (2) vasovagal (tilt-induced) syncope and psychogenic pseudo-syncope represents more than two third of all causes, while brady- and tachyarrhythmic syncope occurs in $25 \%$ of the patients; (3) rhythmic, vasovagal and psychogenic causes of syncope can reasonably be predicted using simple models based on clinical history, age and P-wave duration.

\section{Acknowledgements}

This work was supported by the Fondation Vaudoise de Cardiologie and by the Fondation Suisse de Bourses en Médecine et Biologie. 


\section{References}

[1] Sarasin FP, Louis-Simonet M, Carballo D, et al. Prospective evaluation of patients with syncope: a population-based study. Am J Med 2001;111 (3):177-84.

[2] Ammirati F, Colivicchi F, Santini M. Diagnosing syncope in clinical practice. Implementation of a simplified diagnostic algorithm in a multicentre prospective trial - the OESIL 2 study (Osservatorio Epidemiologico della Sincope nel Lazio). Eur Heart J 2000;21 (11):935-40.

[3] Croci F, Brignole M, Alboni P, et al. The application of a standardized strategy of evaluation in patients with syncope referred to three syncope units. Europace 2002;4(4):351-5.

[4] Ammirati F, Colivicchi F, Minardi G, et al. The management of syncope in the hospital: the OESIL Study (Osservatorio Epidemiologico della Sincope nel Lazio). G Ital Cardiol 1999;29(5):533-9.

[5] Blanc JJ, L'Her C, Touiza A, Garo B, L'Her E, Mansourati J. Prospective evaluation and outcome of patients admitted for syncope over a 1 year period. Eur Heart J 2002;23(10):815-20.

[6] Farwell DJ, Sulke AN. Does the use of a syncope diagnostic protocol improve the investigation and management of syncope? Heart 2004;90 (1):52-8.

[7] Colivicchi F, Ammirati F, Melina D, Guido V, Imperoli G, Santini M. Development and prospective validation of a risk stratification system for patients with syncope in the emergency department: the OESIL risk score. Eur Heart J 2003;24(9):811-9.

[8] Brignole M, Disertori M, Menozzi C, et al. Management of syncope referred urgently to general hospitals with and without syncope units. Europace 2003;5(3):293-8.

[9] Wayne HH. Syncope. Physiological considerations and an analysis of the clinical characteristics in 510 patients. Am J Med 1961;30:418-38.

[10] Calkins H, Shyr Y, Frumin H, Schork A, Morady F. The value of the clinical history in the differentiation of syncope due to ventricular tachycardia, atrioventricular block, and neurocardiogenic syncope. Am J Med 1995;98(4):365-73.

[11] Martin GJ, Adams SL, Martin HG, Mathews J, Zull D, Scanlon PJ. Prospective evaluation of syncope. Ann Emerg Med 1984;13 (7):499-504.

[12] Brignole M, Alboni P, Benditt D, et al. Task force on syncope, European Society of Cardiology. Part 1. The initial evaluation of patients with syncope. Europace 2001;3(4):253-60.

[13] Mittal S, Stein KM, Markowitz SM, Slotwiner DJ, Rohatgi S, Lerman BB. Induction of neurally mediated syncope with adenosine. Circulation 1999;99:1318-24.

[14] Ammirati F, Colivicchi F, Biffi A, Magris B, Pandozi C, Santini M. Head-up tilt testing potentiated with low-dose sublingual isosorbide dinitrate: a simplified time-saving approach for the evaluation of unexplained syncope. Am Heart J 1998;135(4):671-6.

[15] Michelucci A, Bagliani G, Colella A, et al. P wave assessment: state of the art update. Card Electrophysiol Rev 2002;6(3):215-20.

[16] Brignole M, Menozzi C, Bartoletti A, et al. A new management of syncope: prospective systematic guideline-based evaluation of patients referred urgently to general hospitals. Eur Heart J 2006;27(1):76-82.

[17] Farwell DJ, Sulke AN. A randomised prospective comparison of three protocols for head-up tilt test and carotid sinus massage. Int J Cardiol 2005;105(3):241-9.

[18] Sarasin FP, Carballo D, Slama S, Louis-Simonet M. Usefulness of 24-h Holter monitoring in patients with unexplained syncope and a high likelihood of arrhythmias. Int J Cardiol 2005;101(2):203-7.
[19] Sagrista-Sauleda J, Romero-Ferrer B, Moya A, Permanyer-Miralda G, Soler-Soler J. Variations in diagnostic yield of head-up tilt test and electrophysiology in groups of patients with syncope of unknown origin. Eur Heart J 2001;22(10):857-65.

[20] Kapoor WN, Karpf M, Wieand S, Peterson JR, Levey GS. A prospective evaluation and follow-up of patients with syncope. N Engl J Med 1983;309:197-204.

[21] Silverstein MD, Singer DE, Mulley AG, Thibault GE, Barnett GO. Patients with syncope admitted to medical intensive care units. JAMA 1982;248:1185-9.

[22] Oh JH, Hanusa BH, Kapoor WN. Do symptoms predict cardiac arrhythmias and mortality in patients with syncope? Arch Int Med 1999; 159:375-80.

[23] Linzer M, Felder A, Hackel A, et al. Psychiatric syncope: a new look at an old disease. Psychosomatics 1990;31:181-8.

[24] Linzer M, Varia I, Pontinen M, Divine GW, Grubb BP, Estes III NA. Medically unexplained syncope: relationship to psychiatric illness. Am J Med 1992;92(1A):18S-25S.

[25] Kouakam C, Lacroix D, Klug D, Baux P, Marquie C, Kacet S Prevalence and prognostic significance of psychiatric disorders in patients evaluated for recurrent unexplained syncope. Am J Cardiol 2002;89(5):530-5.

[26] Alboni P, Brignole M, Menozzi C, et al. Diagnostic value of history in patients with syncope with or without heart disease. J Am Coll Cardiol 2001;37(7):1921-8.

[27] Moya A, Brignole M, Menozzi C, et al. Mechanism of syncope in patients with isolated syncope and in patients with tilt-positive syncope. Circulation 2001;104(11):1261-7.

[28] Engel GL. Psychologic stress, vasodepressor (vasovagal) syncope, and sudden death. Ann Intern Med 1978;89:403-12.

[29] Brignole M, Menozzi C, Moya A, et al. Mechanism of syncope in patients with bundle branch block and negative electrophysiological test. Circulation 2001;104(17):2045-50.

[30] Alboni P, Brignole M, Menozzi C, et al. Clinical spectrum of neurally mediated reflex syncopes. Europace 2004;6(1):55-62.

[31] Sheldon R, Rose S, Connolly S, Ritchie D, Koshman ML, Frenneaux M. Diagnostic criteria for vasovagal syncope based on a quantitative history. Eur Heart J 2006;27(3):344-50.

[32] Carey BJ, Eames PJ, Panerai RB, Potter JF. Carbon dioxide, critical closing pressure and cerebral haemodynamics prior to vasovagal syncope in humans. Clin Sci (Lond) 2001;101(4):351-8.

[33] Sanders P, Morton JB, Kistler PM, et al. Electrophysiological and electroanatomic characterization of the atria in sinus node disease: evidence of diffuse atrial remodeling. Circulation 2004;109(12):1514-22.

[34] Aytemir K, Aksoyek S, Yildirir A, Ozer N, Oto A. Prediction of atrial fibrillation recurrence after cardioversion by $\mathrm{P}$ wave signal-averaged electrocardiography. Int J Cardiol 1999;70(1):15-21.

[35] Madu EC, Baugh DS, Gbadebo TD, Dhala A, Cardoso S. Effect of ethnicity and hypertension on atrial conduction: evaluation with highresolution P-wave signal averaging. Clin Cardiol 2001;24(9):597-602.

[36] Kenny RA, O'Shea D, Walker HF. Impact of a dedicated syncope and falls facility for older adults on emergency beds. Age Ageing 2002;31(4):272-5.

[37] Shen WK, Decker WW, Smars PA, et al. Syncope Evaluation in the Emergency Department Study (SEEDS): a multidisciplinary approach to syncope management. Circulation 2004;110(24):3636-45. 